diagnosis about 5 years, census data used to identify male residents per birth interval were those published for the following interval (eg, 2005 estimates used for 1996-2000 births).

Results From 1986 to 2000, 321 DBMD males resided in an MD STARnet site during one or more birth intervals, which produced a prevalence of $1 / 5000$ residents. Race/ethnic-specific prevalence tended to be higher for Hispanics than non-Hispanic whites or African-Americans. Prevalence tended to be decreased for the most recent birth interval.

Conclusion Our results are the first U.S. population-based report of race/ethnic-specific DBMD prevalence. Expansion of MD STARnet to two additional sites will permit computing prevalence for additional race/ethnic subgroups and birth intervals.

\section{P2-261 FACTORS ASSOCIATED WITH OVERWEIGHT IN CHILDREN: CASE STUDY IN THE SOUTHERN BRAZIL}

doi:10.1136/jech.2011.142976j.94

${ }^{1} \mathrm{C}$ Rosaneli, ${ }^{*} \mathrm{~F}$ Auler, ${ }^{2} \mathrm{~A}$ Nakashima, ${ }^{2} \mathrm{C}$ Rosaneli, ${ }^{1} \mathrm{M}$ Orlandoski, ${ }^{3} \mathrm{E}$ Netto-Oliveira, ${ }^{3}$ A Oliveira, ${ }^{1,4} \mathrm{~J}$ Faria-Neto. ${ }^{1} P U C P R$, Curitiba, Paraná, Brazil; ${ }^{2} P U C P R$, Maringá, Paraná, Brazil; ' UEM, Maringá, Paraná, Brazil; ${ }^{3}$ Hospital Constantini, Curitiba, Paraná, Brazil

Excess weight in childhood is a predictor of co morbidity in adulthood. This research aimed to identify factors associated with overweight among schoolchildren. This cross-sectional study involved children of both sexes aged between six and 10.9 years of age, enrolled in 24 public and private schools in the urban region of Maringá, Paraná, southern Brazil. The collection were done in the school environment, with measurement of the weight and height defined by calculating the Body Mass Index (Cole et al 2000; 2007). The socioeconomic status of families was considered the guidelines of ABEP (2008). For the statistical analysis it was used the variance analysis model and the $\chi^{2}$ test, considering $p<0.05$. The total evaluation of variables was done getting adjusted to a model of Multinomial Logistic Regression considering the nutritional condition as response variable and the age, gender, socioeconomic status and Body Mass Index as explanatory variables. This project was approved by the Permanent Ethic Committee of Researches involving human beings from UEM. From 5037 schoolchildren, $53.2 \%$ were female, age range $8.7 \pm 1.3$ years old and $24.1 \%$ presented excess weight. Overweight children from private schools and better socio-economic conditions showed positive relation with the excess weight $(p<0.001)$ and children younger than 8 years old have more chances of being overweight $(p=0.038)$. The impact of these results accelerates the urgency of preventive actions towards overweight and its intercorrences in precoce ages.

\section{P2-262 GREEN TEA CONSUMPTION REDUCES CANCER MORTALITY IN JAPAN: THE JICHI MEDICAL SCHOOL COHORT STUDY}

\section{doi:10.1136/jech.2011.142976j.95}

${ }^{1} \mathrm{~T}$ Takeshima, ${ }^{1} \mathrm{~S}$ Ishikawa, ${ }^{2} \mathrm{~T}$ Saegusa, ${ }^{3} \mathrm{~T}$ Ojima, ${ }^{1} \mathrm{Y}$ Nakamura, ${ }^{1} \mathrm{E}$ Kajii. ${ }^{1} \mathrm{Jichi}$ Medical University, Shimotsuke, Tochigi, Japan; ${ }^{2}$ Sakuma Hospital, Tenryu, Hamamtsu, Shizuoka, Japan; ${ }^{3}$ Hamamatsu University School of Medicine, Higashi-ku, Hamamatsu, Japan

Introduction Previous studies have shown that Green tea consumption reduces colon cancer mortality and the risk of liver cancer. However, no studies have examined the association between green tea consumption and all-cancer mortality.

Methods A multi-centre population based prospective cohort study in 12 districts in Japan collected baseline data on 12490 participants from 1992 to 1995. Individuals for whom a history of green team consumption was missing were excluded as were individuals with a past history of any cancer, myocardial infarction and stroke. Green tea consumption was measured using self-report questionnaires. Date and cause of death were determined by death certificates review. Data were analysed using Cox proportional hazards modelling.

Results In total 10197 Japanese adults aged 40-89 years old, 3936 men and 6261 women, were included in the study. Over 11.9 years of follow-up 887 individuals died; 352 from cancer. In men, the hazard of all-cancer mortality relative to those who reported drinking $<1$ cup/day of green tea was 0.50 (95\% CI 0.27 to 0.93 ) for $1-2$ cups/day, 0.75 (0.46 to 1.21 ) for $3-4$ cups/day, 0.61 (0.38 to 0.99 ) for $\geqq 5 \mathrm{cups} /$ day, respectively ( $p=0.22$ for trend). Corresponding values in women were 0.60 (0.29 to 1.20$), 0.59$ (0.34 to 1.02 ), 0.48 ( 0.27 to 0.84 ), respectively ( $p=0.02$ for trend).

Conclusion Green tea consumption is associated with a reduced hazard of all-cancer mortality.

\section{P2-263 EPIDEMIOLOGY OF CHRONIC RENAL FAILURE AMONG ADMITTED PATIENTS TO THE MILITARY HOSPITAL, TAIZ (YEMEN)}

doi:10.1136/jech.2011.142976j.96

J Saleh. ${ }^{*}$ Faculty of Medicine, Aden University, Aden, Yemen

Objective This study was conducted with the principal objective of contributing to the study of the problem of renal failure in Yemen and the factors related to it.

Material and Methods All the files of the patients diagnosed as chronic renal failure being admitted to the military hospital in Taiz Governorate (Yemen) for haemodialysis during the period June-Nov 2010 representing 99 cases from Taiz, Ibb and Hodeidah Governorates were systematic reviewed and statistically analysed using simple percentage.

Results The results showed that the males were affected more than females with a ratio of 6:4 with a peak incidence group above 40 years for both sex, and most of the admitted patients were farmers being all chat chewers (70\%). Malaria was reported among $30 \%$ of the admitted patients while another $30 \%$ of them were having a history of renal paranchymal diseases several years before. Death was reported in $27 \%$ of the cases and the outcome was unknown in most of the patients.

Conclusions High number of cases were reported in the last years in different governorates of Yemen which could be regarded as an alarming situation.

Recommendations An urgent need for further studies to explore deeply the associated factors to this problem for possible interventions actions to control it as well as improving the facilities for renal dialysis in different hospitals.

\section{P2-264 POTENTIAL LIVES SAVED BY CHRONIC DISEASE PREVENTION AND CONTROL IN LATIN AMERICA AND THE CARIBBEAN}

doi:10.1136/jech.2011.142976j.97

${ }^{1}$ A Samuels, * ${ }^{2} \mathrm{~J}$ Hospedales, ${ }^{1} \mathrm{~N}$ Unwin. ${ }^{1}$ University of the West Indies, Cave Hill, Barbados; ${ }^{2}$ Pan American Health Organization, Washington, USA

Introduction Chronic noncommunicable diseases are the major cause of death and disability in all regions of the world, with the exception of sub Saharan Africa, with particularly high levels in parts of the Caribbean and Latin America (LAC). The WHO has shown conservatively that a $2 \%$ reduction per year for 10 years in mortality from chronic noncommunicable diseases is achievable from a combination of population wide measures and targeting individuals at high risk. Our aim was to inform public health priority setting 